Ebisu Ebisu

Études japonaises Études japonaises

47 | printemps-été 2012

Catastrophes du 11 mars 2011, désastre de

Fukushima : fractures et émergences

\title{
Politiques de dédommagement pour les agriculteurs de Fukushima? Le cas de la Nōminren
}

The Politics Surrounding Reparations for Fukushima Farmers: the Case of

Nōminren

福島の農家にとっての損害賠償を巡る政治

Shintarō Namioka

\section{OpenEdition}

Journals

Édition électronique

URL : http://journals.openedition.org/ebisu/387

DOI : 10.4000/ebisu.387

ISSN : 2189-1893

Éditeur :

Institut français de recherche sur le Japon (UMIFRE 19 MAEE-CNRS), Maison franco-japonaise

Édition imprimée

Date de publication : 1 juin 2012

Pagination : 151-163

ISSN : 1340-3656

Référence électronique

Shintarō Namioka, «Politiques de dédommagement pour les agriculteurs de Fukushima? Le cas de la Nōminren », Ebisu [En ligne], 47 | printemps-été 2012, mis en ligne le 03 avril 2014, consulté le 01 mai 2019. URL : http://journals.openedition.org/ebisu/387 ; DOI : 10.4000/ebisu.387 


\section{Politiques de dédommagement pour les agriculteurs de Fukushima?}

\section{Le cas de la Nōminren}

NAMIOKA Shintarō 浪岡新太郎

À la suite des fuites radioactives des centrales nucléaires, l'agriculture du département de Fukushima est en crise. Dans les zones sous surveillance qui ont été évacuées dans un périmètre de 20 kilomètres autour des centrales nucléaires (keikai kuiki 警戒区域), il est désormais officiellement interdit de poursuivre toute activité agricole. Dans les zones situées entre 20 et 30 kilomètres de distance des centrales nucléaires (kinkyūji hinan junbi $k u i k i$ 緊急時避難準備区域), la population doit être prête à se réfugier à tout moment. L'agriculture n'y est pas interdite mais le risque de contamination radioactive des produits agricoles est élevé 1 . Dans les autres zones ${ }^{2}$, le niveau de contamination des produits est souvent inférieur aux taux limites fixés par le gouvernement. Ces taux officiels ne semblent pas crédibles, si

Maître de conférences en sciences politiques à la Faculté des études internationales à l'université Meiji gakuin (Yokohama, Japon). Chercheur associé au laboratoire CHERPA de l'Institut d'études politiques d'Aix-en-Provence.

1. La version initiale de cet article a été rédigée le 29 octobre 2011. Fin octobre, le gouvernement supprimait cette zone spéciale. La question de la sécurité alimentaire reste cependant d'actualité.

2. Il existe aussi une zone où la quantité annuelle de l'irradiation dépasse $20 \mathrm{mSv}$ (keikakuteki hinan kuiki 計画的避難区域). Les habitants n'ont pas été évacués immédiatement, mais il leur est toutefois demandé de prévoir de se réfugier ailleurs. Cette zone s'étend entre 20 et 50 kilomètres des centrales nucléaires. Une partie de la zone entre 20 et 30 kilomètres de distance des centrales nucléaires, ainsi qu'une partie située entre 30 et 50 kilomètres sont donc classées dans cette catégorie. 
on les compare aux taux limites réglementaires bien inférieurs fixés dans d'autres pays ${ }^{3}$.

Selon les contextes dans lesquels ils se trouvent, les agriculteurs à Fukushima doivent parfois faire un choix entre arrêter, suspendre ou poursuivre leurs activités agricoles. Malgré la disparité de leurs choix, tous ces agriculteurs sont d'avis qu'étant donnés les choix difficiles auxquels ils sont confrontés, ils doivent être considérés comme des victimes à part entière et, à ce titre, être indemnisés.

Mais que signifie l'indemnisation pour les agriculteurs ? Rétablir la situation antérieure à la fuite radioactive ? La situation des agriculteurs pratiquant la vente directe sera tout particulièrement traitée ici. En effet, la vente directe pouvait permettre à ces agriculteurs de vendre leurs produits à des prix leur garantissant de meilleurs revenus. Elle leur permettait surtout de s'engager au niveau individuel sur la qualité des produits auprès des clients et de mettre en avant une crédibilité individuelle.

Leurs pertes ne sauraient donc être réduites aux pertes financières; s'y ajoute en effet la perte du rapport de confiance établi avec les clients au cours de ce rapport interpersonnel qu'est la vente directe. L'indemnité peut être demandée individuellement ou collectivement. Mais étant donné la complexité du processus d'indemnisation, souvent les agriculteurs s'impliquent dans les organisations agricoles.

Parmi ces organisations participant aux mouvements réclamant l'indemnisation, la principale organisation agricole est sans doute la Nōgyō kyōdō kumiai 農業協同組合, ou Nōkyō (Japan Agricultural Cooperatives), proche du Parti libéral démocrate. Cependant, en ce qui concerne les agriculteurs en vente directe, le groupe le plus actif à Fukushima est la branche locale de la Nōmin undō zenkoku rengōkai 農民運動全国連合会, ou Nōminren (Fédération nationale des Paysans), proche du parti communiste. La Nōminren est une organisation rassemblant des fermes de petite et moyenne tailles à l'échelle nationale, ce qui différencie aussi la Nōminren de la Nokyō qui regroupe les plus gros producteurs. Elle se singularise

3. Pour les produits alimentaires ordinaires comme le riz, le taux réglementaire est de $500 \mathrm{bq} / \mathrm{kg}$ - soit deux, voire trois fois plus que le taux admis dans les autres pays. [N.D.L.R. : en avril 2012, ce taux a été ramené à 100 bq/kg par le ministère de la Santé.] 
par ses activités très dynamiques sur le plan politique : la Nōminren s'est notamment illustrée dans des mouvements de lutte contre les zones de libre échange, de défense de la vente directe, ou encore de protection de la souveraineté alimentaire du pays ainsi que sur le plan de la sûreté des produits. La Fédération possède ainsi son propre laboratoire testant les taux de pesticides et de radioactivité des produits agricoles et milite pour un usage modéré des pesticides.

Comment la Nōminren arrive-t-elle à introduire cet aspect humain des pertes alors que les discussions sur l'indemnisation sont souvent réduites à l'anonymat des aspects financiers?

Contrairement à la Nōkyō qui fonctionne en tant que mandataire, la Nōminren aide simplement les agriculteurs à établir des demandes individuelles ${ }^{4}$. Pourquoi la Nōminren ne veut-elle pas être mandatée par les agriculteurs ? Le mandat faciliterait beaucoup la procédure et la rapidité des indemnisations.

L'hypothèse proposée est la suivante : dans la formulation de sa demande d'indemnisation, la Nōminren tenterait de susciter des occasions lui permettant non seulement de présenter une demande d'indemnité financière en fonction des dégâts particuliers ${ }^{5}$, mais aussi d'interroger le processus ou la nature de l'indemnisation elle-même, dans un refus de réduire l'indemnisation à l'aspect financier. Pour ce faire, la Nōminren refuse de représenter les agriculteurs afin de donner à chacun d'entre eux l'opportunité de s'exprimer à la fois de façon individuelle, et dans une perspective commune à toute l'humanité.

4. La Nōminren conseille les agriculteurs pour toutes les questions touchant à l'indemnisation et les aide à remplir les documents nécessaires. Cette activité est ouverte à tous, y compris aux non-membres. Mais il faut noter que l'adhésion à la Nōminren est fortement sollicitée. Il semble donc que l'action de conseil fasse bien partie d'une stratégie d'extension de son influence, non sans un certain succès (journal Nömin 農民, 29 janvier 2012).

5. La Nokyō est mandatée par les agriculteurs et tend à proposer un montant uniforme d'indemnisation selon les prix des produits agricoles déterminés par Nōkyo. Mais dans la vente directe, les prix sont souvent déterminés par les particuliers et normalement plus élevés que ceux proposés par la Nōkyo. Présenter individuellement une demande d'indemnisation augmente la possibilité d'être indemnisé à un niveau plus élevé. 
Pour étudier cette hypothèse, nous clarifierons d'abord le processus et les logiques de l'indemnisation des agriculteurs par Tepco, processus et logiques qui tendent à uniformiser la diversité des demandes. Nous examinerons ensuite les critiques formulées par la Nōminren à l'encontre de ce processus. Nous examinerons enfin la diversité des attentes par rapport aux indemnisations des agriculteurs membres de la Nōminren à Fukushima. Et ce, en prenant en compte le facteur géographique (à savoir la zone dans laquelle ils se trouvent, voir supra), pour savoir si la Nōminren suscite effectivement chez les victimes à la fois une diversité de points de vue et d'attentes et une perspective commune.

\section{Le processus et la logique de l'indemnisation uniformisatrice}

\section{Les instances et le processus d'indemnisation}

La loi définissant les procédures d'indemnisation est dite Genshiryoku songai baishō hō 原子力損害賠償法 (ou GBH, loi d'indemnisation des dommages nucléaires). Cette loi prévoit que l'indemnisation débutera une fois l'ampleur des dégâts déterminée. Un système d'indemnisation provisoire a été mis en place dans l'attente d'évaluations définitives : il dépend de la Cellule de gestion des dégâts économiques causés par l'accident nucléaire (Genshiryoku hatsudensho jiko ni yoru keizai higai taisaku honbu 原子力 発電所事故による経済被害対策本部). Ce mécanisme a pour but de permettre aux agriculteurs en difficulté et dans une situation encore fluctuante d'éviter l'endettement si leurs produits ne se vendaient pas correctement.

L'article 3 de la GBH dispose que si les centrales nucléaires du Japon causent des dégâts, ce sont les entreprises en charge de ces centrales qui en assument la responsabilité ; ce sont donc elles qui sont responsables des indemnisations des victimes. L'article 18 prévoit cependant la création d'une Commission sur les propositions contribuant à l'accord sur l'indemnisation entre parties (Genshiryoku songai baishō shinsakai 原子力損害賠償審査会). Toutefois, sans qu'il soit nécessaire d'attendre l'avis de la Commission, ces entreprises responsables peuvent indemniser. Tepco a toutefois attendu l'avis de la Commission, ce qui a beaucoup ralenti les démarches d'indemnisation. Il existe en outre un Centre de résolution des conflits autour des 
questions de l'indemnisation (Genshiryoku songai baishō funsō kaiketsu sentā 原子力損害賠償紛争解決センター) dont le but est d'intervenir dans les cas de désaccord sur l'indemnisation entre les parties pour aboutir à une conciliation.

Les étapes à suivre sont les suivantes :

1. Les personnes qui s'estiment victimes des fuites radioactives demandent à Tepco l'envoi des documents à remplir pour l'indemnisation ;

2. Tepco les leur transmet ;

3. Après les avoir remplis, les victimes les renvoient à Tepco ;

4. Tepco commence alors à les examiner et, si nécessaire, demande des pièces supplémentaires pour constituer le dossier. Les victimes sont alors informées par courrier du montant des dégâts évalués par Tepco ainsi que de l'offre d'indemnisation ;

5. Si les victimes sont d'accord avec la somme proposée, elles communiquent cet accord à Tepco par courrier et sont indemnisées des dégâts constatés. Si les victimes n'acceptent pas cette proposition, elles peuvent avoir recours au centre de résolution des conflits. Si, malgré cela, les victimes et Tepco sont toujours en désaccord, il ne reste plus que le recours en contentieux.

\section{Quelques caractéristiques du processus}

\section{Le processus de détermination des dégâts financiers}

Si nous concentrons notre attention sur les demandes d'indemnisation concernant les produits agricoles, dès le début d'avril 2011, il apparaît que les dégâts dans la zone dite à évacuer (voir supra) ont bien été reconnus comme ayant été causés par les fuites radioactives de façon unanime. Mais dans les autres zones citées ou dans certains cas particuliers, la détermination des dégâts se fait par la négociation.

Par exemple, pour les dégâts causés à l'extérieur des zones d'évacuation indiquées par le gouvernement, et en dehors de la période d'interdiction de vente imposée par les administrations, Tepco demande aux agriculteurs de fournir des preuves indiquant que les dégâts constatés ont bien été causés par la fuite radioactive et non pour le tremblement de terre ou le tsunami. Les agriculteurs doivent apporter la preuve des profits qu'ils auraient réali- 
sés s'il n’y avait pas eu cette fuite, en comparant le bilan de l'année courante avec celui des années précédentes.

\section{Les méthodes du règlement}

Concernant l'indemnité provisoire, Tepco n'accepte de payer à l'avance que la moitié de l'estimation des pertes. Ầ partir du mois d'octobre, cette procédure ne fut plus acceptée, Tepco n'acceptant désormais que les indemnités définitives. Ce versement n'est toutefois effectué que tous les trois mois.

\section{Les rapports humains dans l'indemnisation}

Enfin, il semble important de signaler que les pertes occasionnées en termes de perte du capital confiance pour les producteurs en vente directe ne sont presque pas prises en compte. D'ailleurs, ces pertes ne se réduisent pas à l'aspect financier (Ōshima \& Yokemoto 2012 : 93).

\section{Le positionnement de la Nōminren}

Dès le mois de mars, la Nōminren a commencé à travailler sur les indemnisations des agriculteurs. Le principe général de la Nōminren consiste à demander à Tepco d'indemniser tous les types de dégâts causés par la fuite radioactive en fonction de la situation individuelle de chaque victime.

\section{Critique du processus de détermination des dégâts financiers}

D’une façon plus générale, la Nōminren critique la logique d'indemnisation poursuivie par Tepco (Nemoto 2011a) et principalement les procédures administratives jugées beaucoup trop lourdes. Premièrement, les documents nécessaires à l'évaluation des dégâts (un fascicule d'environ 160 pages et beaucoup de papiers attestant des liens de causalité entre les dégâts et la fuite radioactive) sont trop compliqués à rassembler et cette difficulté risque d'amener les victimes à renoncer à l'indemnisation.

Deuxièmement, Tepco demande aux agriculteurs de bien distinguer les dégâts causés par la fuite nucléaire de ceux causés par le tsunami et le séisme, alors que ces différentes catastrophes sont intimement liées dans beaucoup de cas. 
Enfin, la Nōminren est d'avis que la charge de fournir des preuves, si elle est nécessaire, ne doit pas être assumée par les agriculteurs mais par Tepco puisque cette dernière est responsable de la situation et donc des demandes d'indemnisation.

\section{Critique des méthodes de règlement ${ }^{6}$}

Tepco tend à indemniser très tardivement les victimes qui ont pourtant des obligations financières ${ }^{7}$ (soin des animaux, semences, etc.) ne pouvant être différées. Pour la Nōminren, l'indemnisation doit être payée tous les mois ou à l'avance. Les versements espacés de trois mois ne correspondent pas aux coûts et aux besoins quotidiens des agriculteurs. L'argent nécessaire à la vie quotidienne commence déjà à manquer.

\section{De la demande d'indemnisation centrée sur les pertes en termes de rapports humains à une mobilisation pour un monde sans nucléaire ${ }^{8}$}

Les critiques émises concernant l'indemnisation visent avant tout à faciliter le processus de demande d'indemnisation individuelle et à adapter l'indemnisation aux besoins financiers des individus et non à ceux de Tepco ${ }^{9}$. Mais les critiques de la Nōminren ne se limitent pas à cela.

En tenant compte de la logique individuelle des victimes, la Nōminren essaie de saisir l'indemnisation non seulement en termes de remboursement financier mais aussi par rapport aux choix de vie de chacun. Dans certains cas, la fuite nucléaire a irréversiblement nui à la vie des agriculteurs pour qui les rapports humains (établis ou sans lien avec leur activité agricole) comptent beaucoup plus. Les dégâts causés par la fuite radioactive ont affecté tous les choix de vie malgré la diversité de ces choix.

6. Voir journal Nömin, 30 décembre 2011.

7. Voir journal Nömin, 11 septembre 2011.

8. Voir Nemoto 2011b.

9. D'ailleurs, les critiques de la Nōminren ont abouti à quelques modifications du processus à la faveur des demandeurs individuels, comme la validation du dossier proposé individuellement qui n'est pas constitué suivant le modèle fourni par Tepco (journal Nōmin, 11 décembre 2011). 
La Nōminren considère ces dégâts irréversibles comme une preuve de l'impossibilité de se baser sur l'énergie nucléaire sur le long terme : il faut donc revoir le modèle d'indépendance énergétique du Japon.

De ce point de vue, les fuites nucléaires ne concernent pas seulement les agriculteurs des zones contaminées mais plus globalement nos sociétés dans leur ensemble, dans la mesure où elles soulèvent des questions relatives au développement.

Les agriculteurs membres de la Nōminren se sentent responsables de cette lutte contre le nucléaire. Ayant vécu cette catastrophe irréversible, en mettant en avant leur attachement absolu à ce qu'ils ont perdu définitivement ainsi qu’à la sûreté alimentaire et à un monde sans nucléaire, ils tentent de préserver leur image de producteurs crédibles et de recréer un rapport de confiance avec les clients ${ }^{10}$.

La Nōminren s'engage activement dans les mouvements sociaux favorables à l'abandon des centrales nucléaires en insistant sur l'irréversibilité des dégâts ${ }^{11}$. Leurs demandes d'indemnisation sont donc aussi formulées conformément à cette prise de position. Elle demande à Tepco d'indemniser toutes les sortes de dégâts y compris lorsqu'ils sont irréversibles, ce qui rendrait le coût de l'énergie nucléaire à son niveau réel impossible et démontrerait son absence de rentabilité. Ce faisant, elle essaie de dissuader les habitants des grandes métropoles - qui consomment la plus grande part de l'énergie produite en province par les centrales nucléaires - d'y avoir recours.

Par ailleurs, la Nōminren prend en considération le contexte dans lequel les personnes ou les communautés de certaines régions ont besoin des centrales nucléaires : elle indique que l'installation des centrales nucléaires est liée à la question du sous-développement. Plus exactement, si les centrales de Tepco ont été installées à certains endroits à Fukushima, c'est parce que ces localités semblaient tellement sous-développées que le choix de les construire là s'est s’imposé. Or, la Nōminren indique que ce choix

10. D'après l'entretien que j'ai réalisé avec le président de la Nōminren à Kōriyama, le 15 octobre 2011.

11. Dans presque toutes les grandes manifestations nationales contre les centrales nucléaires la Nōminren est présente (journal Nōmin, 11 octobre 2011). 
ne s'impose pas et qu'il est possible de proposer d'autres réponses au retard économique de ces provinces.

\section{Proposer des lieux de confrontation entre agriculteurs et Tepco ${ }^{12}$}

La Nōminren organise des manifestations, des sit-in ou encore propose à des agriculteurs des rencontres avec des responsables de Tepco en complément du soutien qu'elle apporte lors de la procédure de demande d'indemnisation. Ce sont des occasions pour les agriculteurs de s'exprimer d'une autre manière, le débat ne se réduisant pas à la question des remboursements des dégâts causés par la fuite radioactive. Quels types de demandes ont pu être formulés selon les trois catégories de zones à risque définies par le gouvernement?

\section{Diversité des choix individuels et attentes par rapport aux indemnisations ${ }^{13}$}

\section{Cesser la production agricole}

Monsieur A. est un agriculteur vivant à l'intérieur du périmètre de la zone évacuée et désormais interdite de 20 kilomètres autour des centrales endommagées de Fukushima. Jusqu'en mars 2011, il habitait la ville de Minamisōma 南相馬, mais vit désormais à Kōriyama 郡山 avec sa famille.

Avant que ne se produise la fuite radioactive, il travaillait en vente directe, distribuant lui-même des paniers de légumes à ses clients abonnés. Pour lui, la procédure d'indemnisation financière ne pose pas beaucoup de problèmes. Les dégâts à indemniser sont bien reconnus dans le cadre des mesures du gouvernement, ses terres étant situées dans une zone identifiée comme étant sinistrée.

12. Pour les manifestations organisées par la Nōminren, voir Nōmin 2011.

13. Ces trois entretiens ont eu lieu à Fukushima, le 15 octobre 2011. Ces cas n'entendent pas être représentatifs des agriculteurs des trois zones, mais sont exemplaires des particularités de chaque zone. 
Parmi les dégâts qui lui semblent difficiles à réparer financièrement, il évoque la difficulté de retrouver un rapport de confiance avec ses clients. Il souffre de la perte ou de la déstabilisation des rapports humains qu'il avait établis avec le temps.

Il veut reprendre l'agriculture, mais étant donné le niveau élevé de radioactivité à Fukushima, il pense reprendre une exploitation dans un lieu plus éloigné géographiquement et donc sans risque de contamination. Mais il souhaite rester à Fukushima pour compter (même un peu) sur le rapport avec des clients fidélisés.

\section{Suspendre l'activité}

Examinons le cas de Monsieur B., éleveur bovin et cultivateur de riz en vente directe à l'intérieur de la zone des 20 à 30 kilomètres dans laquelle on peut résider mais où l'on doit se préparer à pouvoir se réfugier immédiatement, qui reste seul à Minamisōma, sa famille étant réfugiée à Niigata.

Avant le 11 mars, il possédait 55 vaches. Pendant presque deux mois, mars et avril 2011, il a gardé sa ferme malgré le manque d'essence et de fourrage. Pendant ce laps de temps, il n’a pas réussi à vendre son lait suspecté de contamination. Il a finalement décidé de vendre 37 de ses vaches. Depuis le 10 juin 2011, il a recommencé à commercialiser son lait.

Pour lui, l'indemnisation financière n'est pas facile à obtenir. En effet, la zone dans laquelle il exerce est théoriquement exploitable, même si une évacuation d'urgence doit toujours être envisagée. La production laitière n'étant pas interdite dans cette zone, les fermiers sont contraints de produire du lait pour être indemnisés. Sinon, en vertu de l'obligation de fournir des preuves de leurs pertes, ils ne pourront pas comparer le bilan de cette année à celui des années précédentes. Il en est de même pour le riz. Depuis seulement cette année, il a cessé la plantation sur les conseils de la Nōkyō, cet arrêt semblant reconnu par Tepco en termes d'indemnisation.

Concernant les dégâts qui lui semblent difficiles à dédommager financièrement, il évoque le capital de confiance par rapport à la sécurité alimentaire des produits agricoles, même si les niveaux de radioactivité de ses produits sont bien inférieurs aux taux réglementaires. Monsieur B. pense que ce n'est pas possible de continuer son activité dans les conditions antérieures à la fuite radioactive, alors qu'il souhaiterait continuer son travail dans cette région à laquelle il est très attaché. 
Et il considère la décontamination définitive de toutes les zones comme étant impossible, alors que le gouvernement insiste sur l'efficacité du travail de décontamination mené.

\section{Poursuivre l'activité}

Monsieur C. vit à l'extérieur des 30 kilomètres règlementés en zones. Il s'est vu temporairement interdire d'expédier des produits issus de cette zone (Monsieur C. demeure dans la ville de Fukushima).

Il vend du lait pasteurisé en vente directe. Après la fuite radioactive, le lait fut interdit de commercialisation pendant environ 50 jours et il avait du mal à poursuivre l'activité fermière en raison des coupures d'électricité ainsi que du manque de carburant. Les gens commencèrent à se montrer suspicieux quant à la qualité de ses produits et surtout de son lait. Le nombre de ses clients s'est réduit mais pas de manière irréversible. S'agissant de ces pertes, les preuves ne sont pas difficiles à fournir. Quant aux dégâts qui lui semblent difficiles à dédommager financièrement, il évoque le capital sûreté des produits. Il est en tout cas déterminé à continuer son activité comme avant. Comme Monsieur B., il ne fait pas confiance aux critères gouvernementaux et insiste sur la nécessité de procéder à une décontamination totale même si cela est difficile, particulièrement dans les montagnes où il habite.

\section{En guise de conclusion}

La demande d'indemnisation structurée par la Nōminren a permis aux agriculteurs de s'exprimer individuellement sur le montant de dégâts qui leur semblait pertinent et sur les dégâts difficiles à indemniser financièrement, tel que le rapport de confiance avec les clients. Cette demande a deux effets. D'abord, elle insiste sur l'irréversibilité des dégâts. Ensuite, elle établit que ce ne sont pas les agriculteurs qui doivent assumer la responsabilité de la sûreté des produits, élément indispensable dans les rapports de confiance avec les clients. Les taux réglementaires de radioactivité sur lesquels l'indemnisation repose ne leur semblent pas crédibles étant donné les taux fixés à l'étranger. De fait, les agriculteurs essaient de garantir la sûreté alimentaire eux-mêmes, alors que c'est le gouvernement ou Tepco qui sont 
responsables : les agriculteurs n'ont pas eux-mêmes créé cette situation dans laquelle ils se voient contraints d'être sensibles au niveau de radioactivité ${ }^{14}$.

Toutefois, cela ne les dispense pas d'être responsables en tant que parties prenantes du modèle de développement actuel fondé sur les centrales nucléaires. C'est pourquoi les agriculteurs de la Nōminren demandent l'abandon des centrales nucléaires dans le cadre de la demande d'indemnisation, pour que des dégâts similaires ne soient plus jamais causés. Cette demande n'est pas seulement exprimée par les agriculteurs en tant que victimes directement concernées mais au nom de l'humanité toute entière.

Compte tenu de la complexité du processus d'indemnisation et des procédures juridiques, compte tenu aussi de ce que la question de la contamination radioactive repose sur des expertises de différentes sortes, les agriculteurs en tant que " profanes » ont du mal à s'exprimer au cours de ce processus. Ils risquent de ne pas s'exprimer ou d'être représentés de manière uniforme alors qu'ils constituent des parties prenantes aux intérêts différents. Nous pourrions considérer que la lutte pour l'indemnisation menée par la Nōminren consiste à générer un espace contre-public subalterne permettant aux agriculteurs de s'exprimer individuellement mais également dans une perspective commune, en leur fournissant les connaissances nécessaires leur permettant de s'affirmer dans ce processus ${ }^{15}$. Cet espace qui prend la forme d'une demande individuelle accompagnée par la Nōminren fonctionne comme un espace de délibération dans lequel les agriculteurs (re)définissent leurs besoins, les dégâts causés et leurs responsabilités. Même si l'aspect exclusif de cet espace est patent au sens où une pression est exercée sur les non-membres afin qu'ils adhèrent et cotisent à la Nōminren, il s'agit

14. Pour une critique de la structure sociale qui a produit cet incident, voir Ōshima et Yokemoto (2012).

15. Le concept d'espace public est utilisé comme un espace où les citoyens en tant qu'individus délibèrent autour d'une perspective commune. S’il n'y a qu'un seul espace public, cet espace tend nécessairement à refouler des opinions qui ne correspondent pas à ses critères. Il faut toujours envisager ce concept d'espace public au pluriel, ainsi que leurs interactions dans la conflictualité autour de la définition légitime du public. Fraser qualifie de " faibles " les espaces publics exerçant peu d'influence sur la décision politique, comme les espaces contre-publics subalternes (Fraser 1997, Dryzeck 2002). Les mérites du concept d'espace public ne consistent pas à produire l'accord d'une façon unanime, mais à mettre en lumière la pluralité des opinions et la delibération qui l'accompagnent. 
de rendre possible, dans cet espace, le questionnement sur l'indemnisation définie par le gouvernement et par Tepco. Il s'agit aussi de reconnaître les disparités des demandes individuelles tout en défendant le bien commun. C'est un cas particulier de démocratie délibérative dans un espace contrepublic subalterne, souvent difficilement organisée par des citoyens ordinaires, autour d'une question technique dans laquelle les citoyens sont parties prenantes au premier chef.

29 octobre 2011

\section{Bibliographie}

DRYZECK John, 2002

Deliberative Democracy and Beyond,

Oxford, Oxford University Press.

FRASER Nancy, 1997

Justice Interruptus, Londres, Routledge.

NEMOTO Satoshi 根本敬, 2011a

«Fukushima kara Fukushima, soshite Fukushima e » 福島からフクシマ、そして FUKUSHIMAへ (Fukushima :

du lieu japonais à l'événement universel, en passant par l'objet médiatique), Nōmin, juillet, 64 : 20-29.

\section{NEMOTO Satoshi, 2011b}

« Jishin, tsunami, genpatsu jiko kara no saisei »地震·津波·原発事故からの再生 (Le renouveau après le tremblement de terre, le tsunami et l'accident nucléaire), Buraku mondai kenkyū 部落問題研究， septembre, $820: 77-83$.

ŌSHIMA Ken.ichi 大島堅一

\& YOKEMOTO Masafumi 除本理史, 2012 Genpatsu jiko no higai to hoshō 原発事故 の被害と補償 (Les dommages nucléaires et les indemnisations), Tokyo, Ōtsuki shoten 大月書店. 